\title{
Effects of Leptonic Nonunitarity on Lepton Flavor Violation, Neutrino Oscillation, Leptogenesis, and Lightest Neutrino Mass
}

\author{
Gayatri Ghosh (iD) and Kalpana Bora \\ Department Of Physics, Gauhati University, Guwahati, Assam 781014, India \\ Correspondence should be addressed to Gayatri Ghosh; gayatrighsh@gmail.com
}

Received 19 January 2018; Revised 20 March 2018; Accepted 2 April 2018; Published 25 June 2018

Academic Editor: Theocharis Kosmas

Copyright (c) 2018 Gayatri Ghosh and Kalpana Bora. This is an open access article distributed under the Creative Commons Attribution License, which permits unrestricted use, distribution, and reproduction in any medium, provided the original work is properly cited. The publication of this article was funded by SCOAP ${ }^{3}$.

\begin{abstract}
Neutrino physics is a mature branch of science with all the three neutrino mixing angles and two mass squared differences determined with high precision. In spite of several experimental verifications of neutrino oscillations and precise measurements of two mass squared differences and the three mixing angles, the unitarity of the leptonic mixing matrix is not yet established, leaving room for the presence of small nonunitarity effects. Deriving the bounds on these nonunitarity parameters from existing experimental constraints, on cLFV decays such as $\mu \rightarrow e \gamma, \mu \rightarrow \tau \gamma$, and $\tau \rightarrow e \gamma$, we study their effects on the generation of baryon asymmetry through leptogenesis and neutrino oscillation probabilities. We consider a model where see-saw is extended by an additional singlet $S$ which is very light but can give rise to nonunitarity effects without affecting the form on see-saw formula. We do a parameter scan of a minimal see-saw model in a type I see-saw framework satisfying the Planck data on baryon to photon ratio of the Universe, which lies in the interval $5.8 \times 10^{-10}<Y_{B}<6.6 \times 10^{-10}(B B N)$. We predict values of lightest neutrino mass and Dirac and Majorana CP-violating phases $\delta_{C P}, \alpha$, and $\beta$, for normal hierarchy and inverted hierarchy for one-flavor leptogenesis. It is worth mentioning that all these four quantities are unknown yet, and future experiments will be measuring them.
\end{abstract}

\section{Introduction}

Neutrinos have nonzero masses. There are 3 known flavors of neutrinos, $v_{e}, v_{\mu}$, and $v_{\tau}$, each of which couples only to the charged lepton of the same flavor. $v_{e}, v_{\mu}$, and $v_{\tau}$ are superpositions of three mass eigenstates, $\left|v_{\alpha}>=\sum U_{\alpha i}^{*}\right| v_{i}>$, where $\alpha=e, \mu, \tau$ and $\nu_{i}$ is the neutrino of definite mass $m_{i}$. The cosmological constraints of the sum of the $\nu$ masses bound are $\sum_{i} m\left(v_{i}\right)<0.23 \mathrm{eV}$ from CMB, Planck 2015 data (CMB15+ LRG+ lensing $+H_{0}$ ) [1]. We note that the lepton mixing matrix $U$ has a big mixing and we know almost nothing about the phases. The discoveries of neutrino mass and leptonic mixing have come from the observation of neutrino flavor change, $v_{\alpha} \rightarrow v_{\beta}$. CP violation interchanges every particle in a process by its antiparticle. This $\mathrm{CP}$ violation can be produced by the phase $\delta_{C P}$ in $U$. Neutrinos can have two types of mass term in the Lagrangian-Dirac and Majorana mass terms. To determine whether Majorana masses occur in nature, so that $\overline{v_{i}}=v_{i}$, the favorable approach to seek is Neutrinoless Double Beta Decay $(0 \nu \beta \beta)$.
In the conventional type I see-saw framework, there are Dirac and Majorana mass matrices $m_{D}$ and $M_{R}$ in the Lagrangian:

$$
L=\frac{1}{2} \overline{N_{R}} M_{R} N_{R}^{c}+N_{R} m_{D} v_{L}+\text { h.c. }
$$

The low-energy mass matrix is given by

$$
m_{v}=-m_{D}^{T} M_{R}^{-1} m_{D}
$$

In the usual unitarity scenario, the three active neutrinos, the flavor eigenstates $v_{e}, v_{\mu}$, and $\nu_{\tau}$ are connected to the mass eigenstates $v_{1}, v_{2}$, and $v_{3}$ via $v_{\alpha}=N_{\alpha i} \nu_{i}$, where $N^{\dagger} N=1$. Here $N$ is the generalized neutrino mixing matrix which could be either unitary or nonunitary. In the diagonal charged lepton basis, $m_{\nu}$ is diagonalized by a unitary matrix as

$$
U P m_{v} P^{\dagger} U^{\dagger}=m_{v}^{D}
$$


The Pontecorvo-Maki-Nakagawa-Sakata (PMNS) matrix is $\mathrm{UP}$, where $\mathrm{U}$ is

$$
=\left(\begin{array}{ccc}
c_{12} c_{13} & s_{12} c_{13} & s_{13} e^{-i \delta} \\
-s_{12} c_{23}-c_{12} s_{23} s_{13} e^{i \delta} & c_{12} c_{23}-s_{12} s_{23} s_{13} e^{i \delta} & s_{23} c_{13} \\
s_{12} s_{23}-c_{12} c_{23} s_{13} e^{i \delta} & -c_{12} s_{23}-s_{12} c_{23} s_{13} e^{i \delta} & c_{23} c_{13}
\end{array}\right) .
$$

Here $\theta_{12}=33.56^{\circ}, \theta_{23}=41.6^{\circ}\left(50^{\circ}\right), \theta_{13}=8.46^{\circ}\left(8.49^{\circ}\right)[3]$ (see [3] for recent global fit values) are the solar, atmospheric, and reactor angles for Normal Ordering (Inverted Ordering), respectively. The Majorana phases reside in $\mathrm{P}$, where

$$
P=\operatorname{diag}\left(\begin{array}{lll}
1 & e^{i \alpha} & e^{i(\alpha+\beta)}
\end{array}\right)
$$

Cosmologists suggest that, just after the Big Bang, the Universe contained equal amounts of matter and antimatter. Today the Universe contains matter but almost no antimatter. This change needs that matter and antimatter act differently (CP violation). The CP-violating scenario to explain this change is leptogenesis. Leptogenesis is a natural outcome of the see-saw mechanism. In the see-saw picture, we assume that, just as there are 3 light neutrinos $v_{1}, v_{2}, v_{3}$, there are 3 heavy right-handed neutrinos $M_{1}, M_{2}, M_{3}$, where $M_{R} \sim$ $10^{9-14} \mathrm{GeV}, M_{R} \sim M_{1}, M_{2}, M_{3}$ which were there in the Hot Big Bang. The $M_{R}$ decays modes are

$$
\begin{aligned}
& M \longrightarrow l^{-}+H^{+}, \\
& M \longrightarrow l^{+}+H^{-}, \\
& M \longrightarrow v+H^{0}, \\
& M \longrightarrow \bar{v}+\overline{H^{0}},
\end{aligned}
$$

where $l^{-}$are $e^{-}, \mu^{-}, \tau^{-}$and $H^{+}, H^{-}, H^{0}$ are SM Higgs. CP violation effects in the $M_{R}$ decays may result from phases in the decay coupling constants. This leads to unequal numbers of leptons $\left(l^{-}\right.$and $\left.\nu\right)$ and antileptons $\left(l^{+}\right.$and $\left.\bar{\nu}\right)$ in the Universe:

$$
\Gamma\left(M \longrightarrow l^{+}+H^{-}\right) \neq \Gamma\left(M \longrightarrow l^{-}+H^{+}\right) .
$$

In leptogenesis, CP-violating decays of heavy Majorana neutrinos create a lepton-antilepton asymmetry [4] and then $\mathrm{B}+\mathrm{L}$ violating sphaleron processes [5] at and above the electroweak symmetry breaking scale convert part of this asymmetry into the observed baryon-antibaryon asymmetry. The heavy neutrinos are see-saw partners of the observed light ones.

Depending on mass of the lightest heavy RH Majorana neutrinos (whose decay causes leptogenesis) the leptogenesis can be of three types: unflavored (or one-flavor), twoflavored, and three-flavored leptogenesis. For unflavored leptogenesis, valid for $M_{1} \geq 10^{11} \mathrm{GeV}$, we have taken here $M_{1} \sim$ $10^{12} \mathrm{GeV}$ where the flavor of the final state leptons plays no role. It can be shown that for lower values of $M_{1}$ it depends on the flavor of the final state leptons and hence is called flavored leptogenesis [6]. Here we consider unflavored leptogenesis.
For unflavored leptogenesis, the decay asymmetry in the case of hierarchical heavy neutrinos is given by [7]

$$
\varepsilon_{1}=\frac{1}{8 \pi v^{2}} \frac{1}{\left(m_{D} m_{D}^{\dagger}\right)_{11}} \sum_{2,3} \operatorname{Im}\left(m_{D} m_{D}^{\dagger}\right)_{1 j}^{2} f\left(\frac{M_{j}^{2}}{M_{1}^{2}}\right)
$$

where $f(x)=-3 / 2 \sqrt{x}$ for $x>1$, i.e., for hierarchical heavy neutrinos. The baryon asymmetry of the Universe is proportional to the decay asymmetry $\varepsilon_{1}$. The Dirac mass matrix $m_{D}$ in terms of a complex and orthogonal matrix $R$ is [8]

$$
m_{D}=i \sqrt{M_{R}} R \sqrt{m_{v}^{d i a g}} U^{\dagger}
$$

The compatible quantity for leptogenesis is then

$$
\begin{aligned}
m_{D} m_{D}^{\dagger} & =\sqrt{M_{R}} R \sqrt{m_{v}^{d i a g}} U^{\dagger} U \sqrt{m_{v}^{\text {diag }}} R^{\dagger} \sqrt{M_{R}} \\
& =\sqrt{M_{R}} R m_{v}^{d i a g} R^{\dagger} \sqrt{M_{R}}
\end{aligned}
$$

If $R$ is real then there is no leptogenesis at all. Here, we have taken $R=U$ (see (4)). $R$ consists of the low-energy mixing elements and the CP phases. Since unitarity of neutrino mixing matrix has not been proved yet, if it is nonunitary, then, for the neutrino mixing matrix $\mathrm{N}$ to be nonunitary, we have

$$
v_{\alpha}=N_{\alpha i} \nu_{i}
$$

connecting the flavor and mass states. The nonunitary matrix $\mathrm{N}$ can be written as

$$
N=(1+\eta) U_{0}
$$

where $U_{0}=U * P$. If $m_{\nu}$, which is diagonalized by a nonunitary mixing matrix, originates from the see-saw mechanism, we have

$$
m_{D}=i \sqrt{M_{R}} R \sqrt{m_{v}^{d i a g}} N^{\dagger}
$$

And, thereupon, we have

$$
m_{D} m_{D}^{\dagger}=\sqrt{M_{R}} R \sqrt{m_{\nu}^{\text {diag }}} N^{\dagger} N \sqrt{m_{\nu}^{\text {diag }}} R^{\dagger} \sqrt{M_{R}} .
$$

For nonunitary $U_{P M N S}$ matrix, $N^{\dagger} N=1+2 U_{0}^{\dagger} \eta U_{0} \neq 1$. Leptogenesis is no longer independent of the low-energy phases. It depends on the phases in $U_{0}$ as well as the phases in $\eta$. Leptogenesis [9] is one of the exceedingly wellinspired frameworks which produces baryon asymmetry of the Universe through $\mathrm{B}+\mathrm{L}$ violating electroweak sphaleron process $[5,10]$.

In the conventional type I see-saw mechanism, due to the mixing of the left-handed and right-handed neutrinos the PMNS matrix is nonunitary. Nevertheless this nonunitarity is too small to have any observable effects in lepton flavor violation (LFV) or neutrino oscillation (see $[7,11,12])$. If we want to connect the lepton mixing matrix to leptogenesis via the Casas-Ibarra parametrization, then there should not be 
any significant sizeable contribution to $m_{v}$ and leptogenesis other than the usual see-saw terms. Hence we must decouple the origin of unitary violation from these terms. Mixing of the light neutrinos with new physics creates nonunitarity in the low-energy mixing matrix. We consider here a model as used in [7] where the see-saw mechanism is enlarged by an additional singlet sector, which leads to a $9 \times 9$ mass matrix. Here

$$
L=\frac{1}{2}\left(\begin{array}{lll}
\overline{v_{L}^{c}} & \overline{N_{R}} & \bar{X}
\end{array}\right)\left(\begin{array}{ccc}
0 & m_{D}^{T} & m^{T} \\
m_{D} & M_{R} & 0 \\
m & 0 & M_{s}
\end{array}\right)\left(\begin{array}{c}
v_{L} \\
N_{R}^{c} \\
X^{c}
\end{array}\right),
$$

where the upper left block is the one corresponding to usual type I see-saw mechanism. It can be diagonalized with a unitary matrix $F$, such that

$$
F^{T}\left(\begin{array}{ccc}
0 & m_{D}^{T} & m^{T} \\
m_{D} & M_{R} & 0 \\
m & 0 & M_{s}
\end{array}\right) F=\left(\begin{array}{ccc}
m_{v}^{\text {diag }} & 0 & 0 \\
0 & M_{R} & 0 \\
0 & 0 & M_{s}^{\text {diag }}
\end{array}\right)
$$

The form of $F$ is as given in [7]. As discussed in [7] with proper choice of various elements in $F$ and of $M_{s}$, it can be shown that

$$
m_{\nu}^{\text {diag }}=-\widetilde{N^{T}} m_{\nu}^{D} M_{R}^{-1} m_{\nu}^{D} \widetilde{N}
$$

which shows that $N=\left(\widetilde{N^{\dagger}}\right)^{-1}$ is the lepton mixing matrix, as there is no other significant contribution to the mass term of the light neutrinos. Thus in this model the usual see-saw mechanism remains unaltered with unmodified leptogenesis. Here $M_{R}$ does not couple to the new singlets but a sizeable nonunitary lepton mixing matrix $N$ can be induced, thus providing us with a framework where we can apply (14).

We consider here that lepton asymmetry is generated by out-of-equilibrium decay of heavy right-handed Majorana neutrinos into Higgs and lepton within the framework of type I see-saw mechanism. In a hierarchical case of three right-handed heavy Majorana neutrinos $M_{2,3}>M_{1}$, with the lepton asymmetry created by the decay of $M_{1}$, the lightest one of three heavy right-handed neutrinos is [13]

$$
\begin{aligned}
\varepsilon_{1}^{\alpha} & =\frac{1}{8 \pi v^{2}} \\
& \cdot \frac{1}{\left(m_{D}^{\dagger} m_{D}\right)_{11}}\left[\sum_{2,3} \operatorname{Im}\left[\left(m_{D}^{*}\right)_{\alpha 1}\left(m_{D}^{\dagger} m_{D}\right)_{1 j}\left(m_{D}\right)_{\alpha j}\right]\right. \\
& \cdot g\left(x_{j}\right)+\sum_{2,3} \operatorname{Im}\left[\left(m_{D}^{*}\right)_{\alpha 1}\left(m_{D}^{\dagger} m_{D}\right)_{j 1}\left(m_{D}\right)_{\alpha j}\right] \\
& \left.\cdot \frac{1}{1-x_{j}}\right]
\end{aligned}
$$

Here $v=174 \mathrm{GeV}$ is the vacuum expectation value of the SM Higgs and $g(x)=\sqrt{x}(1+1 /(1-x)-(1+x) \ln ((1+x) / x))$, where $x_{j}=M_{j}^{2} / M_{1}^{2}$.
At temperatures, $T \geq 10^{12} \mathrm{GeV}$, all the charged leptons are in equilibrium because the direct and inverse decays are very frequent and wash out any asymmetry. At moderate temperatures $T<10^{12} \mathrm{GeV}\left(T<10^{9} \mathrm{GeV}\right)$, some particles decouple and thus flavor effects play an important role in the calculation of lepton asymmetry $[6,14-18]$. The regions of temperatures belonging to $10^{9}<\mathrm{T} / \mathrm{GeV}<10^{12}$ and $\mathrm{T} / \mathrm{GeV}<10^{9}$ are, respectively, denoted as two and three flavor regimes of leptogenesis [19].

The building blocks of matter are the quarks, the charged leptons, and the neutrinos. The discovery and study of the Higgs boson at the Large Hadron Collider (LHC) have provided strong evidence that the quarks and charged leptons derive their masses from a coupling to the Higgs field. Most theorists strongly believe that the origin of the neutrino masses is different from the origin of the quark and charged lepton masses. Neutrino oscillation has proved that neutrinos have nonzero masses. We and all matter may have descended from heavy neutrinos. We list the values of $m_{\text {lightest }}$ for oneflavor for different hierarchies and unitarity and nonunitarity of $U_{P M N S}$ in Table 2 and check whether our values of $m_{\text {lightest }}$ are consistent with the constraints on the absolute scale of $v$ masses. The new results presented in this work are as follows:

(i) We have calculated new values of nonunitarity parameters of $U_{P M N S}$ matrix from the bounds on rare cLFV decays.

(ii) Hence we predicted the absolute value of lightest $v$ mass in this regard. The values of lightest $v$ mass lie in the ranges of $0.0018 \mathrm{eV}$ to $0.0023 \mathrm{eV}, 0.048 \mathrm{eV}$ to $0.056 \mathrm{eV}$, $0.05 \mathrm{eV}$ to $0.054 \mathrm{eV}$, and $0.053 \mathrm{eV}$ to $0.062 \mathrm{eV}$ in one-flavor leptogenesis regime.

(iii) All these values satisfy the constraint $\sum_{i} m\left(v_{i}\right)<$ $0.23 \mathrm{eV}$.

(iv) The predicted values of CP-violating phases, $\delta_{C P}$, and Majorana phases $\alpha$ and $\beta$ are $36^{\circ}, 72^{\circ}, 108^{\circ}, 144^{\circ}, 180^{\circ}$, $216^{\circ}, 252^{\circ}, 288^{\circ}, 324^{\circ}$, and $360^{\circ}$. Here the calculated values of each of them, i.e., the Dirac CP-violating phase, $\delta_{C P}$, and the Majorana phases $\alpha$ and $\beta$, are found to be same.

The paper is organized as follows. In Section 2, we show the effect of low-energy phenomenology of nonunitarity on charged lepton flavor violating decays in type I see-saw theories and present the values of various parameters used in our analysis for the generation of baryon asymmetry of the Universe through the mechanism of leptogenesis. Section 3 contains our calculations and results. Section 4 contains analysis and discussions. Section 5 summarizes the work.

\section{Low-Energy Phenomenology of Nonunitarity and Leptogenesis}

One interesting feature of nonunitarity of the PMNS matrix can be studied in rare charged lepton flavor violation (LFV) decay processes. In the light of unitarity violation in decays such as $\alpha \rightarrow \beta \gamma,(\alpha, \beta)=(\tau, \mu),(\tau, e)$ or $(\mu, e)$, the branching ratio is [7]

$$
\frac{B R(\alpha \rightarrow \beta+\gamma)}{B R(\alpha \rightarrow \beta+\nu \bar{\nu})}=\frac{100 \alpha}{96 \pi}\left|\left(N N^{\dagger}\right)_{\alpha \beta}\right|^{2} .
$$


TABLE 1: Our calculated constraints on nonunitarity parameters $\eta_{\tau e}, \eta_{\tau \mu}$, and $\eta_{\mu e}$ using branching ratios of latest cLFV decays taken from [2].

\begin{tabular}{lcc}
\hline Serial No. & $\begin{array}{c}\text { Latest updated Branching Ratios } \\
\text { on cLFV Decays }\end{array}$ & $\begin{array}{c}\text { Calculated bounds } \\
\text { on }|\eta|_{\alpha \beta}\end{array}$ \\
\hline 1 & $B R(\mu \rightarrow e+\gamma)=4.2 \times 10^{-13}$ & $\left|\eta_{\mu e}\right|=6.64733013 \times 10^{-6}$ \\
2 & $B R(\tau \rightarrow \mu+\gamma)=4.4 \times 10^{-8}$ & $\left|\eta_{\tau \mu}\right|=5.11766 \times 10^{-3}$ \\
3 & $B R(\tau \rightarrow e+\gamma)=3.3 \times 10^{-8}$ & $\left|\eta_{\tau e}\right|=7.021 \times 10^{-3}$ \\
\hline
\end{tabular}

Also

$$
\begin{aligned}
& \frac{B R(\tau \rightarrow \mu+\gamma)}{B R(\tau \rightarrow \mu+\nu \bar{\nu})} \simeq \frac{25 \alpha}{6 \pi}\left|\eta_{\mu \tau}\right|^{2} \\
& \frac{B R(\tau \rightarrow \mu+\gamma)}{4.2 \times 10^{-10}} \simeq \frac{\left|\eta_{\mu \tau}\right|^{2}}{25 \times 10^{-8}}
\end{aligned}
$$

Using the latest updated constraint on $B R(\tau \rightarrow \mu+\gamma)=4.4 \times$ $10^{-8}$ [2], one can derive bounds on $\left|\eta_{\mu \tau}\right|$ from (23). It can be shown that

$$
\frac{B R(\tau \rightarrow \mu+\gamma)}{B R(\mu \rightarrow e+\gamma)}=\frac{B R\left(\tau \rightarrow \mu+\nu_{\tau} \overline{\nu_{\mu}}\right)}{B R\left(\mu \rightarrow e+\nu_{\mu} \overline{\nu_{e}}\right)} \times \frac{\left|\eta_{\mu \tau}\right|^{2}}{\left|\eta_{\mu e}\right|^{2}}
$$

Now, we calculate the ratio

$$
\frac{B R\left(\tau \rightarrow \mu+\nu_{\tau} \overline{\nu_{\mu}}\right)}{B R\left(\mu \rightarrow e+\nu_{\mu} \overline{\nu_{e}}\right)}=0.176745
$$

Thus we find constraints on $\left|\eta_{\mu e}\right|$ from (24), using the latest constraint on $B R(\mu \rightarrow e+\gamma)$, where $B R(\mu \rightarrow e+\gamma)=4.2 \times$ $10^{-13}[2]$. Again we have

$$
\frac{B R(\tau \rightarrow \mu+\gamma)}{B R(\tau \rightarrow e+\gamma)}=\frac{B R\left(\tau \rightarrow \mu+\nu_{\tau} \overline{\nu_{\mu}}\right)}{B R\left(\tau \rightarrow e+\nu_{\mu} \overline{\nu_{e}}\right)} \times \frac{\left|\eta_{\mu \tau}\right|^{2}}{\left|\eta_{\tau e}\right|^{2}}
$$

From our calculation, the ratio $B R\left(\tau \rightarrow \mu+\nu_{\tau} \overline{\nu_{\mu}}\right) / B R(\tau \rightarrow$ $\left.e+\nu_{\mu} \overline{\nu_{e}}\right)$ is

$$
\frac{B R\left(\tau \rightarrow \mu+\nu_{\tau} \overline{\nu_{\mu}}\right)}{B R\left(\tau \rightarrow e+\nu_{\mu} \overline{\nu_{e}}\right)}=2.509
$$

And then we calculate the latest updated bounds on $\left|\eta_{\tau e}\right|$. The calculations are summarized in Table 1. The study of the effects of leptonic nonunitarity on two- and three-flavored leptogenesis is presented in [20]. We note that interesting results on cLFV $\mu \rightarrow e \gamma$ in NUSM, NUHM, NUGM, and mSUGRA models are presented in [21] in which we have predicted some values of new SUSY particles that may be detected at next run of LHC.

Returning to leptogenesis, the baryon asymmetry should lie in the interval $5.8 \times 10^{-10}<Y_{B}<6.6 \times 10^{-10}$ [22]. In general, we have taken complex and orthogonal matrix $R=$ $U_{P M N S}$, but $\mathrm{R}$ can be taken as $R=V_{C K M} \times U_{P M N S}$ in nonSUSY SO(10) models [18] (see [18] for detailed discussion) as studied in the context of breaking entanglement of octant of $\theta_{23}$ and $\delta_{C P}$ in the light of baryon asymmetry of the Universe through the mechanism of leptogenesis.

For the normally ordered light neutrino masses, we have

$$
\begin{aligned}
M_{R}^{\text {diag }} & =\operatorname{diag}\left(M_{1}, M_{2}, M_{3}\right)=M_{1} \operatorname{diag}\left(1, \frac{M_{2}}{M_{1}}, \frac{M_{3}}{M_{1}}\right) \\
& =M_{1} \operatorname{diag}\left(1, \frac{m_{1}}{m_{2}}, \frac{m_{1}}{m_{3}}\right),
\end{aligned}
$$

with $m_{1} \in\left[10^{-6} \mathrm{eV}, 10^{-1} \mathrm{eV}\right]$, and $m_{2}^{2}-m_{1}^{2}=7.60 \times$ $10^{-5} \mathrm{eV}^{2}, m_{3}^{2}-m_{1}^{2}=2.48 \times 10^{-3} \mathrm{eV}^{2}$ as is evident from the neutrino oscillation data [3], with $m_{1}$ being the lightest one of three neutrino masses. For the inverted ordered light neutrino masses, we have

$$
\begin{aligned}
M_{R}^{\text {diag }} & =\operatorname{diag}\left(M_{1}, M_{2}, M_{3}\right)=M_{1} \operatorname{diag}\left(1, \frac{M_{2}}{M_{1}}, \frac{M_{3}}{M_{1}}\right) \\
& =M_{1} \operatorname{diag}\left(1, \frac{m_{1} * m_{3}}{m_{2}^{2}}, \frac{m_{1}}{m_{2}}\right),
\end{aligned}
$$

with $m_{3}$ being the lightest one of the three neutrino masses. Next, we do the parameter scan for one-flavored leptogenesis of a minimal see-saw model satisfying the Planck data on baryon to photon ratio of the Universe for four cases:

(i) Normal hierarchical structure neutrino masses, nonunitarity of PMNS matrix

(ii) Normal hierarchical structure neutrino masses, unitarity of PMNS matrix

(iii) Inverted hierarchical structure of neutrino masses, nonunitarity of PMNS matrix

(iv) Inverted hierarchical structure neutrino masses, unitarity of PMNS matrix.

We perform random scan of the parameter space for $\mathrm{NH}$ and $\mathrm{IH}$ in the light of recent ratio of the baryon to photon density bounds $5.8 \times 10^{-10}<\eta_{B}<6.6 \times 10^{-10}$ in the following ranges:

$$
\begin{aligned}
m_{1}\left(m_{3}\right) & \in\left[10^{-6} \mathrm{eV}, 0.1 \mathrm{eV}\right]\left(\left[10^{-6} \mathrm{eV}, 0.1 \mathrm{eV}\right]\right), \\
\delta_{C P} & \in[0,2 \pi] \\
\alpha & \in[0,2 \pi] \\
\beta & \in[0,2 \pi] .
\end{aligned}
$$

While doing parameter scan, we find values of lightest neutrino mass, Majorana phases $\alpha, \beta$, and Dirac CPV phase $\delta_{C P}$, for which baryon to photon ratio $Y_{B}$ lies in the given range, for above four cases. This is done for oneflavor/unflavored leptogenesis regime. 


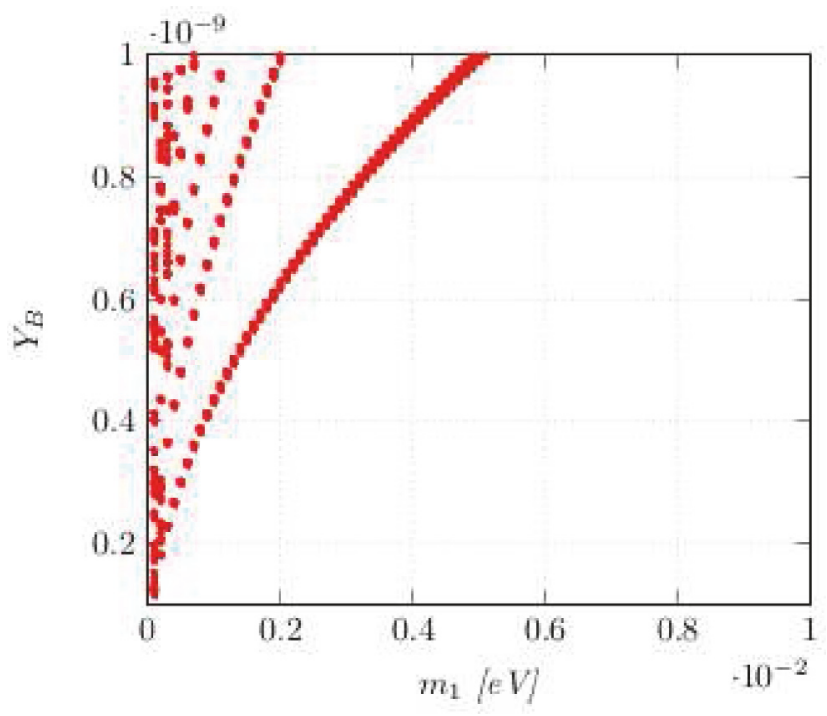

FIGURE 1: Scatter plot of the lightest neutrino mass $m_{1}$ against the baryon asymmetry of the Universe with normal hierarchy, nonunitarity case in one-flavor leptogenesis regime. The values of $m_{1}[\mathrm{eV}]$ along the $\mathrm{x}$-axis are multiplied by $10^{-2}$.

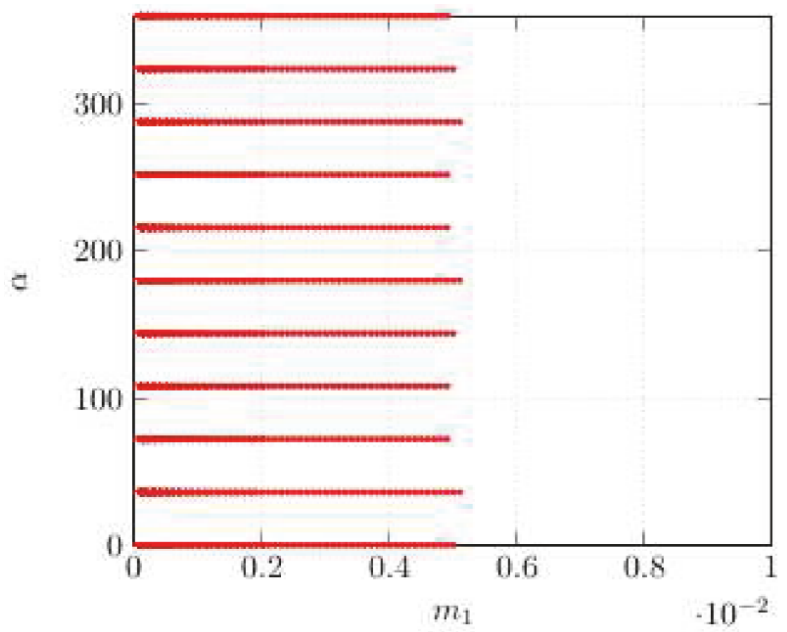

(a)

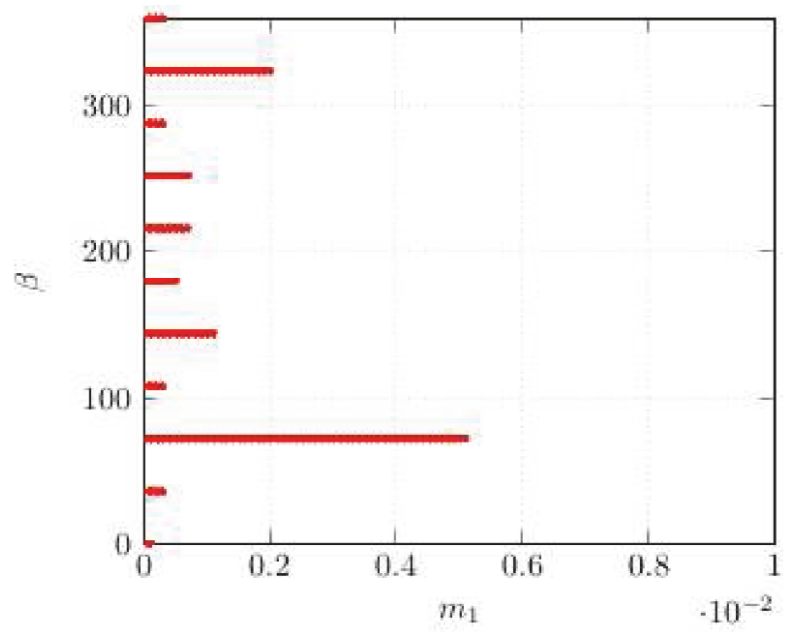

(b)

Figure 2: Variation of lightest neutrino mass $m_{1}$ with Majorana phases $\alpha$ and $\beta$ in case of $\mathrm{NH}$, nonunitarity case in one-flavor leptogenesis regime. $m_{1}$ is in $\mathrm{eV}$. The values of $m_{1}[\mathrm{eV}]$ in (a) and (b) along the $\mathrm{x}$-axis are multiplied by $10^{-2}$.

\section{Calculations and Results}

Results of our analysis have been presented in Figures 1-9. It can be seen from Figure 1 that in the one-flavor regime $\mathrm{NH}$ structure of neutrino masses and nonunitarity textures of PMNS matrix can give rise to correct baryon asymmetry of the Universe, $5.8 \times 10^{-10}<Y_{B}<6.6 \times 10^{-10}$, if the lightest $v$ mass lies around $0.0018 \mathrm{eV}$ to $0.0023 \mathrm{eV}$. A close numerical inspection of the situation reveals that for a lightest neutrino mass of $0.0024 \mathrm{eV}-0.005 \mathrm{eV}$ one can exceed the upper bound on $Y_{B}$. The dependence of lightest neutrino mass $m_{1}$ on Majorana phases $\alpha, \beta$ is shown in (a), (b) of Figure 2, respectively, $Y_{B}$ being constrained in the order $10^{-10}$.
We find that $M_{1}=10^{12} \mathrm{GeV}$ is favored in the light of baryon asymmetry of the Universe for one-flavor regime.

Figure 3 shows the scatter plot of the lightest neutrino mass $m_{1}$ against the baryon asymmetry of the Universe with Normal hierarchy and unitary $U_{P M N S}$ in one-flavor regime. For $Y_{B}$ to be in the range, $5.8 \times 10^{-10}<\eta_{B}<6.6 \times 10^{-10}, m_{1}$ lies between $0.048 \mathrm{eV}$ and $0.056 \mathrm{eV}$. For $Y_{B}$ in the order $10^{-10}$, the lightest neutrino mass $m_{1}$ is mostly concentrated in the region $0.043 \mathrm{eV}$ to $0.06 \mathrm{eV}$.

In Figure 4 we have shown the variation of lightest neutrino mass $m_{1}$ with Dirac CP phase $\delta_{C P}$ and Majorana phase $\alpha$ for $\mathrm{NH}$ (normal hierarchy) and unitarity texture. For $Y_{B}$ to be in the consistent BAU range $5.8 \times 10^{-10}<Y_{B}<6.6 \times 10^{-10}$, 


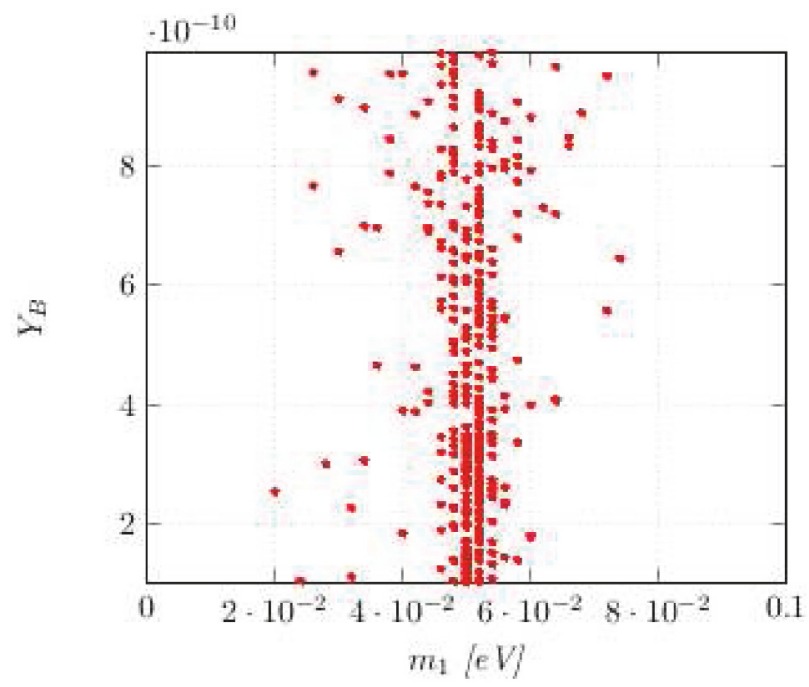

FIGURE 3: Scatter plot of the lightest neutrino mass $m_{1}$ against the baryon asymmetry of the Universe with normal hierarchy, unitarity case in one-flavor leptogenesis regime.

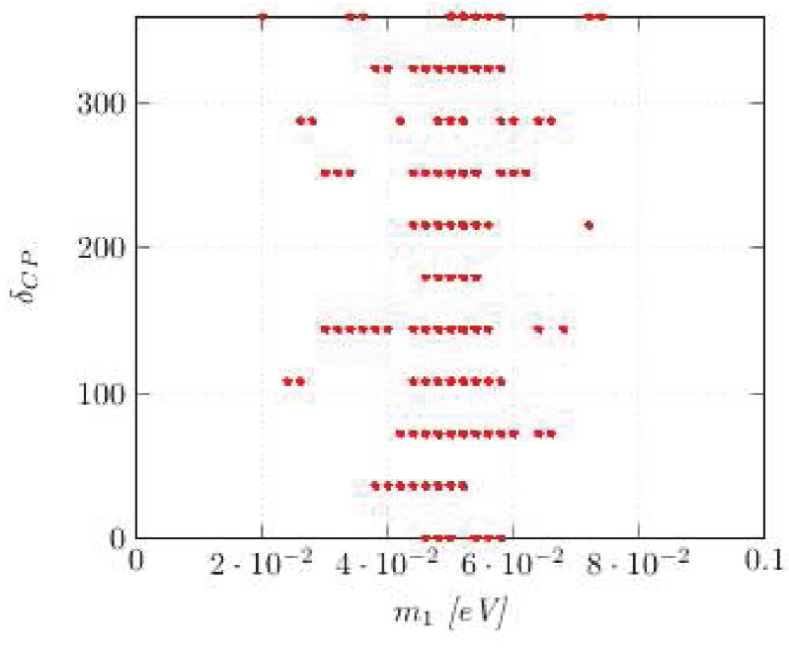

(a)

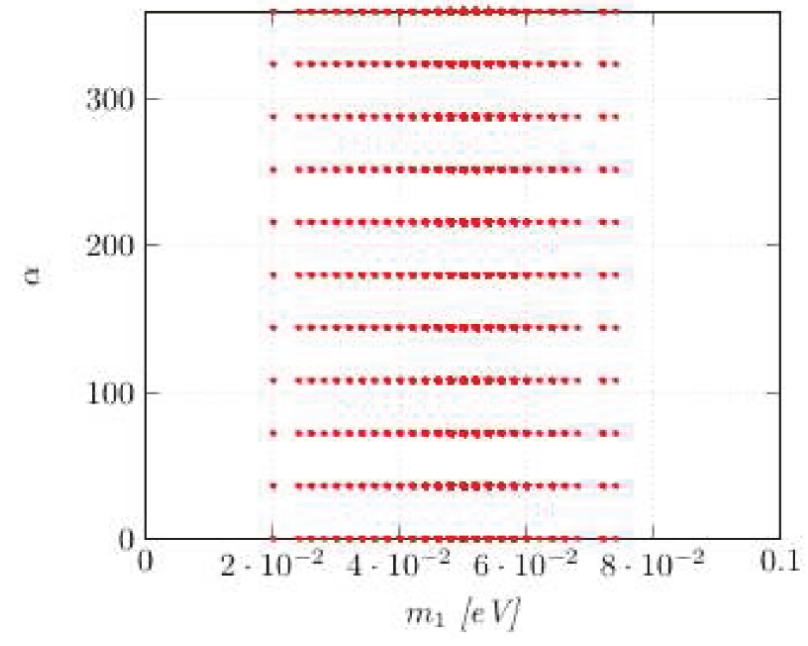

(b)

Figure 4: Variation of lightest neutrino mass $m_{1}$ on Dirac CP phase $\delta_{C P}$ and Majorana phases $\alpha$ in case of NH unitarity case in one-flavor leptogenesis regime.

one of the values of $\delta_{C P}$ predicted by us, i.e., $\delta_{C P}=252.9^{\circ}$ is also favored in the recent global fit values, $\delta_{C P}=253.8^{\circ}$ for normal hierarchy. It can be seen from Figure 5 that, in the one-flavor regime, $\mathrm{IH}$ structure of neutrino masses and nonunitarity textures of PMNS matrix can give rise to baryon asymmetry of the Universe, of the order of $10^{-10}$, if the lightest neutrino mass lies around $0.05 \mathrm{eV}$ to $0.054 \mathrm{eV}$. Few points lie in the region, $5.8 \times 10^{-10}<Y_{B}<6.6 \times 10^{-10}$.

Figure 6 shows the scattered plot of lightest neutrino mass $m_{3}$ against Dirac CPV phase $\delta_{C P}$ and Majorana phase $\alpha$ in IH of neutrino mass and nonunitarity case of oneflavor leptogenesis regime. Figure 7 shows the variation of the lightest neutrino mass against the baryon asymmetry of the Universe and Dirac CP phase $\delta_{C P}$ with inverted hierarchy and unitarity case in one-flavor leptogenesis regime. For $Y_{B}$ in the order $10^{-10}$, the lightest $\nu$ mass $m_{3}$ is concentrated in the region $0.053 \mathrm{eV}$ to $0.062 \mathrm{eV}$. In Figure 8 we have shown the scatter plot of $m_{3}$ with Majorana phases $\alpha$ and $\beta$ for IH and unitarity texture of $U_{P M N S}$ in one-flavor leptogenesis regime.

In Figure 9, we have shown the effect of nonunitarity on probability $P\left(\nu_{\mu} \rightarrow v_{e}\right)$ for a particular case of Long Baseline Neutrino Experiments (DUNE, FNAL, USA). We have used the value of baseline to be $\mathrm{L}=1300 \mathrm{Km}$ and values of other oscillation parameters are taken from latest global fit [3]. It can be seen that nonunitarity affects the probability, which means that its effect could be studied in neutrino oscillation experiments provided we reach the required precision level. 


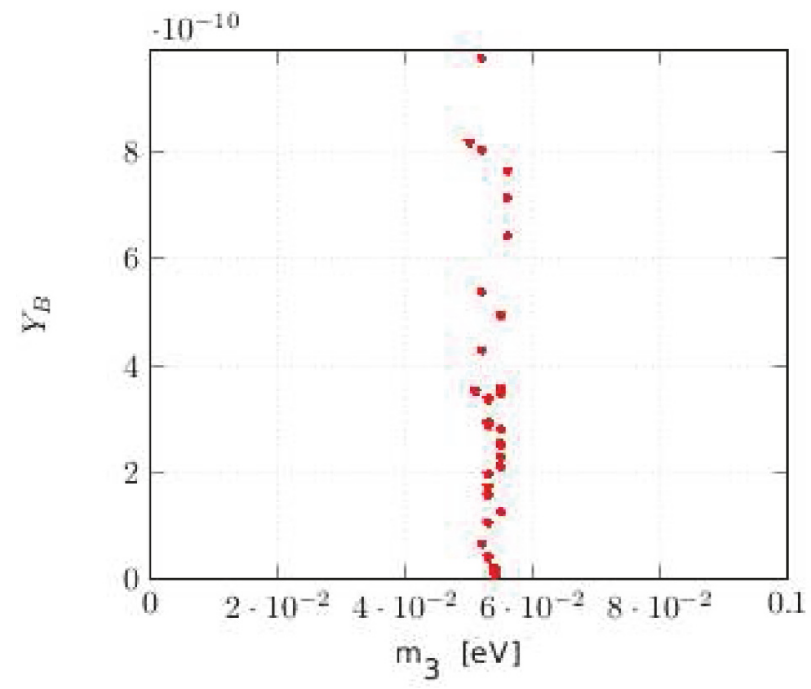

FIGURE 5: Scatter plot of the lightest neutrino mass $m_{3}$ against the baryon asymmetry of the Universe with inverted hierarchy, nonunitarity case in one-flavor leptogenesis regime.

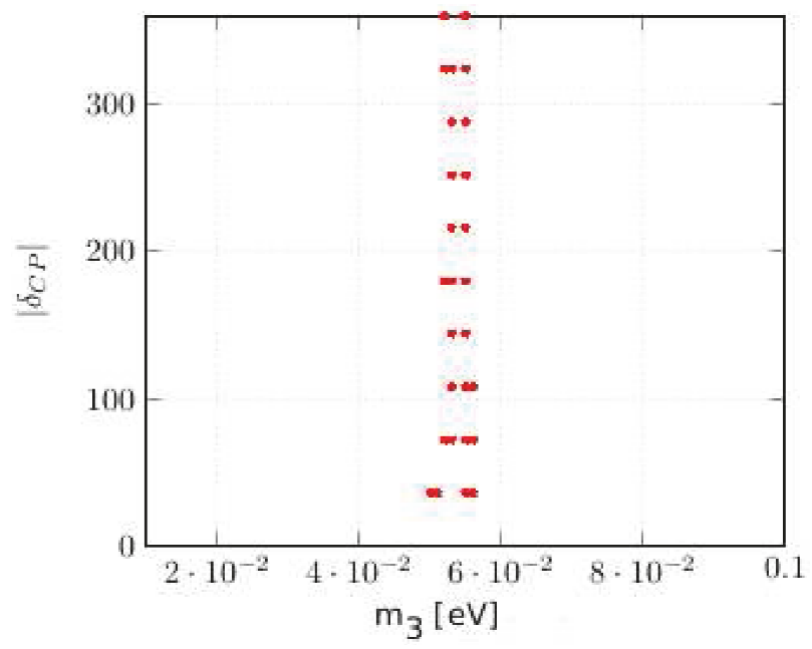

(a)

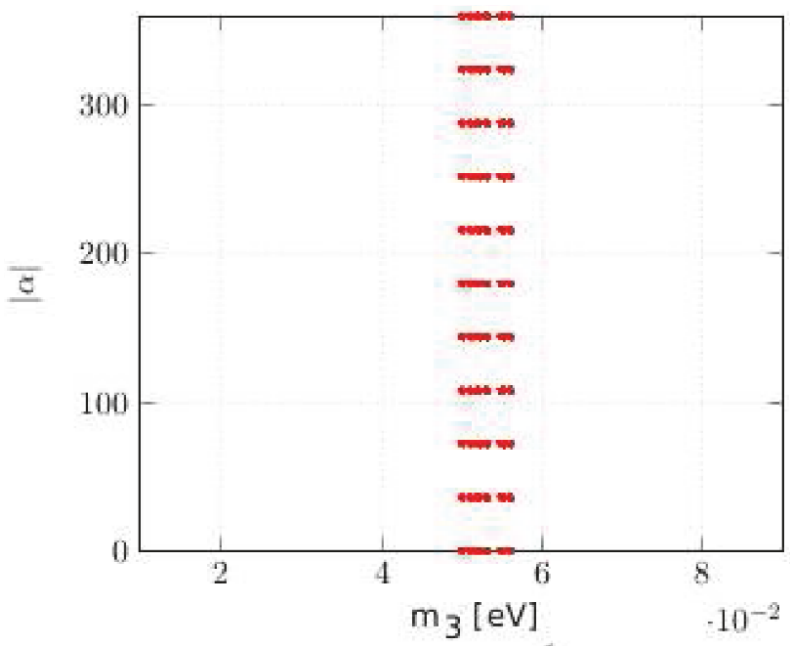

(b)

Figure 6: Variation of lightest neutrino mass $m_{3}$ against Dirac CP phase $\delta_{C P}$ and Majorana phase $\alpha$ in case of IH, nonunitarity case in one-flavor leptogenesis regime. The values of $m_{3}[\mathrm{eV}]$ in (b) along the $\mathrm{x}$-axis are multiplied by $10^{-2}$.

\section{Analysis and Discussion}

We present here some comments that reflect the main inference of this work. From the results presented in Table 2 we see that in all the four cases there exist values of lightest neutrino mass, satisfying the constraint $\sum_{i} m\left(v_{i}\right)<0.23 \mathrm{eV}$ and those of present day baryon asymmetry of the Universe. The analysis of our results can be summarized as follows:

(i) One-flavor leptogenesis: The value of lightest neutrino mass shifts to higher value in $\mathrm{IH}$ case, as compared to $\mathrm{NH}$. Nonunitarity effects decrease the value of lightest neutrino mass in both $\mathrm{NH}$ and $\mathrm{IH}$ and the diminishing effect is more severe in $\mathrm{NH}$.

(ii) We also found that nonunitarity affects the probability of $\nu$ oscillation $P\left(\nu_{\mu} \rightarrow \nu_{e}\right)$.
TABLE 2: Our calculated results for $m_{\text {lightest }}$ with inverted hierarchy, normal hierarchy, and one-flavor leptogenesis. The symbol $\checkmark(x)$ is used when $Y_{B}$ is within (not within) updated BAU range.

\begin{tabular}{lcc}
\hline Case & $m_{\text {lightest }}$ & One Flavor \\
\hline $\mathrm{NH}$, non-unitarity & $0.0018 \mathrm{eV}$ to $0.0023 \mathrm{eV}$ & $\checkmark$ \\
$\mathrm{NH}$, unitarity & $0.048 \mathrm{eV}$ to $0.056 \mathrm{eV}$ & $\checkmark$ \\
IH, non-unitarity & $0.05 \mathrm{eV}$ to $0.054 \mathrm{eV}$ & $\checkmark$ \\
IH, unitarity & $0.053 \mathrm{eV}$ to $0.062 \mathrm{eV}$ & $\checkmark$ \\
\hline
\end{tabular}

(iii) The predicted values of CP-violating phases, $\delta_{C P}$, and Majorana phases $\alpha$ and $\beta$ are $36^{\circ}, 72^{\circ}, 108^{\circ}, 144^{\circ}, 180^{\circ}$, $216^{\circ}, 252^{\circ}, 288^{\circ}, 324^{\circ}$, and $360^{\circ}$. Here the calculated values of 


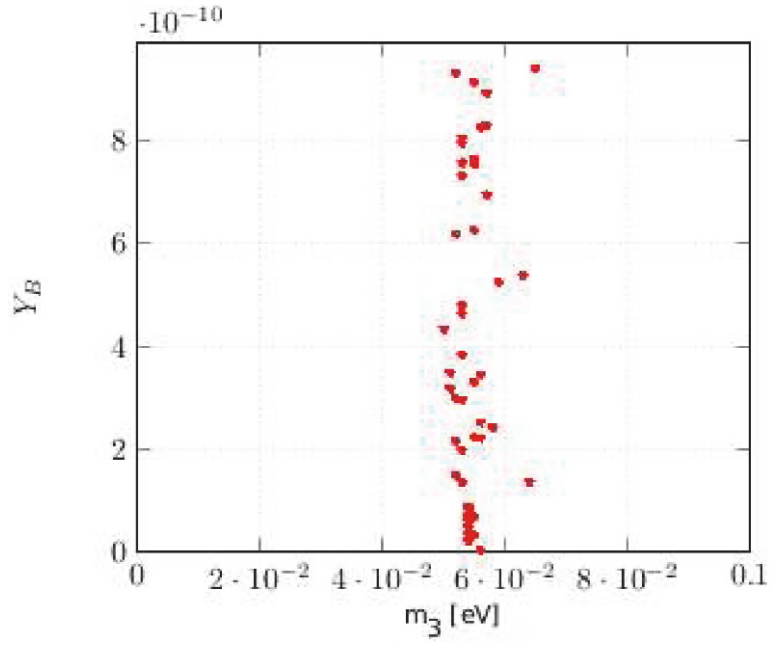

(a)

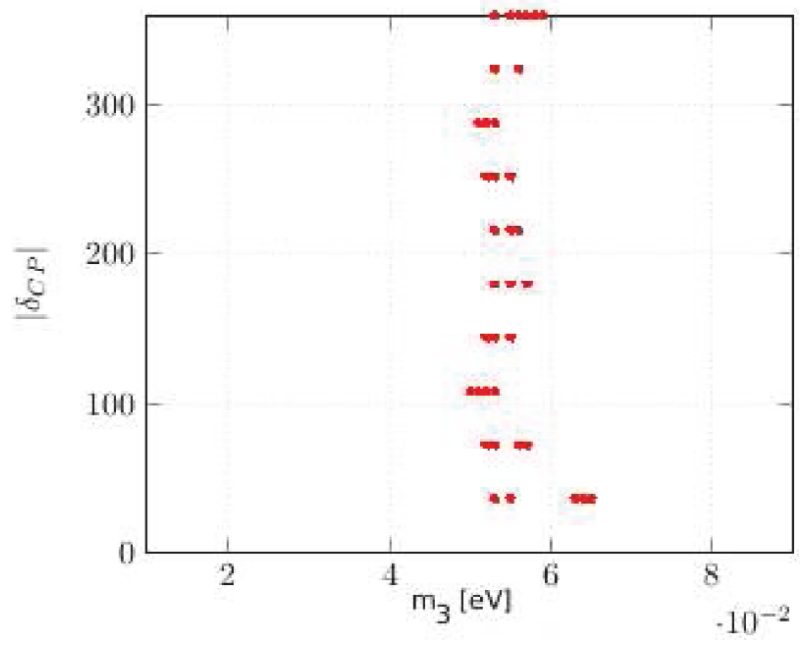

(b)

Figure 7: Scatter plot of the lightest neutrino mass against the baryon asymmetry of the Universe and Dirac CP phase $\delta_{C P}$ with inverted hierarchy, unitarity case in one-flavor leptogenesis regime. For $Y_{B}$ in the order $10^{-10}$, the lightest $\nu$ mass $m_{3}$ is concentrated in the region $0.053 \mathrm{eV}$ to $0.062 \mathrm{eV}$. The values of $m_{3}[\mathrm{eV}]$ in (b) along the $\mathrm{x}$-axis are multiplied by $10^{-2}$.

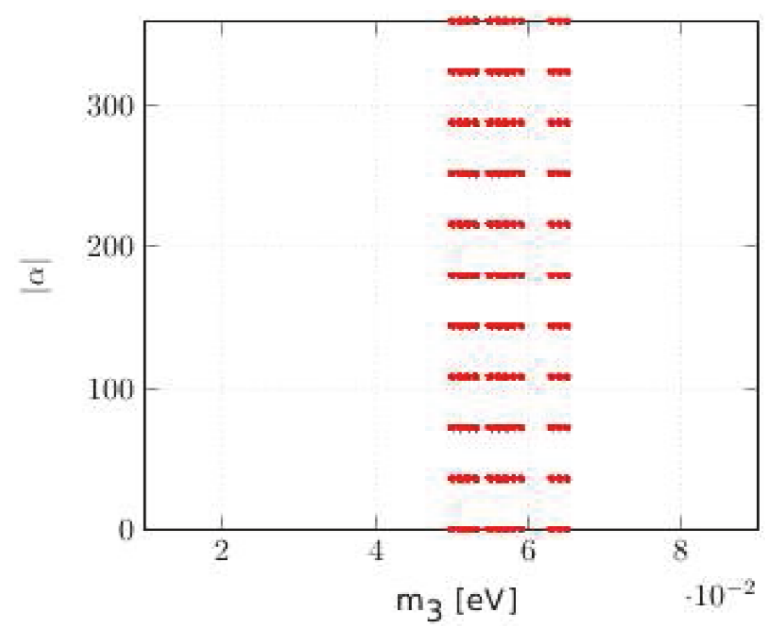

(a)

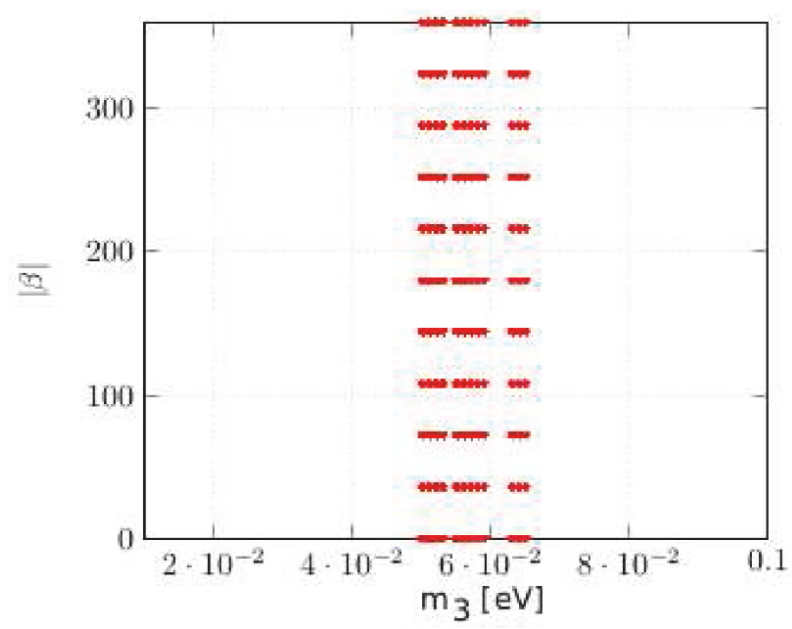

(b)

FIGURE 8: Variation of lightest neutrino mass $m_{3}$ with Majorana phases $\alpha$ and $\beta$ for IH, unitarity texture of $U_{P M N S}$ in one-flavor leptogenesis regime. The values of $m_{3}[\mathrm{eV}]$ in (a) along the $\mathrm{x}$-axis are multiplied by $10^{-2}$.

each of the Dirac CP-violating phase, $\delta_{C P}$, and the Majorana phases $\alpha$ and $\beta$ are found to have the same set of values, i.e., $36^{\circ}, 72^{\circ}, 108^{\circ}, 144^{\circ}, 180^{\circ}, 216^{\circ}, 252^{\circ}, 288^{\circ}, 324^{\circ}$, and $360^{\circ}$.

\section{Conclusion}

In conclusion, in this work, we have considered the possibility that the neutrino mixing matrix (considering charged lepton mass matrix to be diagonal), $U_{P M N S}$, could be nonunitary and then calculated the limits on nonunitary parameters $\eta_{\mu e}, \eta_{\tau e}$, and $\eta_{\tau \mu}$ (see Table 1) from latest constraints on branching ratios of cLFV decays. It is well known that in usual type I see-saw mechanism, mixing of left- and righthanded neutrinos may lead to nonunitarity but it has been found that [11] its effect is not significant for processes like lepton flavor violation and neutrino oscillation. Therefore we consider here a model (see [7]) where see-saw is extended by an additional singlet (very light) which, although inducing nonunitarity of the $U_{P M N S}$ matrix, leaves formula for see-saw mechanism unmodified. This nonunitarity however may affect leptogenesis. Baryogenesis through leptogenesis is believed to be responsible for producing the matterantimatter asymmetry present in the present day Universe, which can be expressed through parameter $Y_{B}$ (baryon to photon ratio). We then analysed how the nonunitarity of $U_{P M N S}$ can affect leptogenesis and hence calculated the values of lightest $\nu$ mass, Dirac CPV phase $\delta_{C P}$, and Majorana phases $\alpha$ and $\beta$, such that $Y_{B}$ lies in the present day constraints 


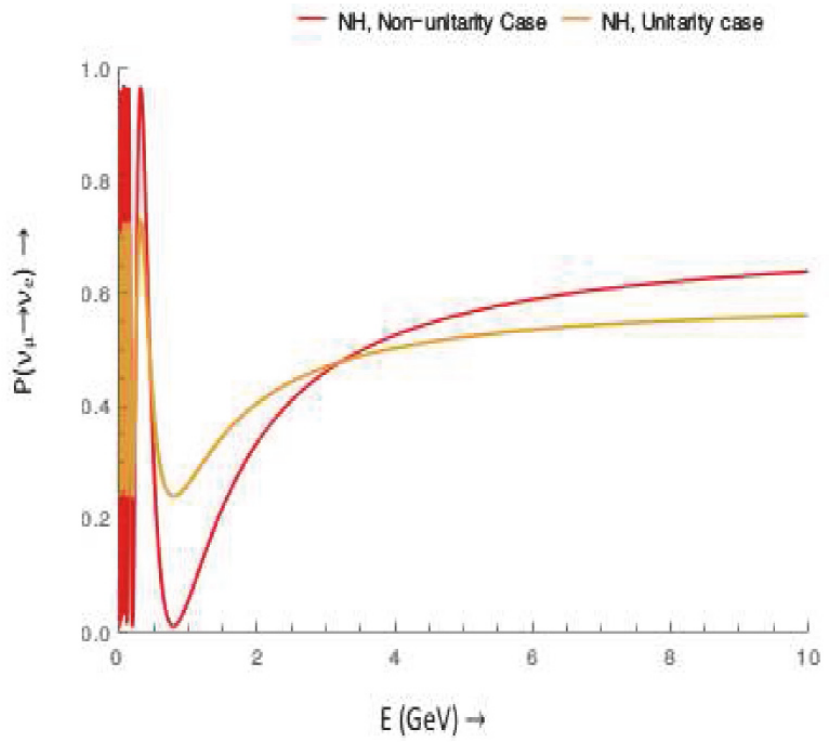

FIGURE 9: Variation of $P\left(\nu_{\mu} \rightarrow v_{e}\right)$ against energy E in Long Baseline Neutrino Experiments with value of Dirac CP phase, $\delta_{C P}=1.45 \pi$, equal to the best fit value [3]. The yellow (red) curve in the figure corresponds to unitarity (nonunitarity) of PMNS matrix.

TABLE 3: The results for Dirac CPV phase $\delta_{C P}$ and two Majorana phases $\alpha, \beta$ of all the four cases mentioned above in one flavor leptogenesis regime in this work. Here the calculated values of each of them (the Dirac CP-violating phase, $\delta_{C P}$, and the Majorana phases $\alpha$ and $\beta$ ) are found to have the same set of values, i.e., $36^{\circ}, 72^{\circ}, 108^{\circ}$, $144^{\circ}, 180^{\circ}, 216^{\circ}, 252^{\circ}, 288^{\circ}, 324^{\circ}$, and $360^{\circ}$.

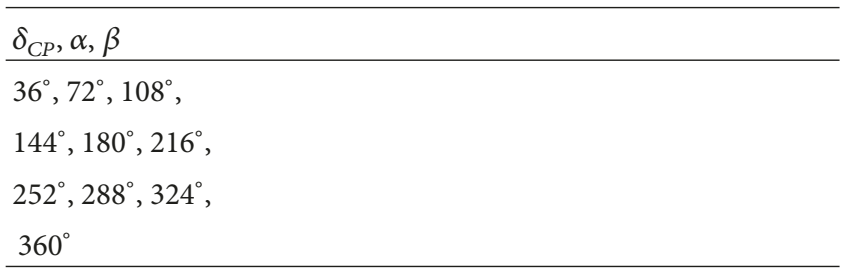

$\left(5.8 \times 10^{-10}<Y_{B}<6.6 \times 10^{-10}\right)$. This was done using type I see-saw mechanisms for producing light neutrino masses.

Above analysis was done for different cases: $\mathrm{NH}$ neutrino masses, unitary $U_{P M N S} ; \mathrm{NH}$ neutrino masses, nonunitary $U_{P M N S}$; IH neutrino masses, unitary $U_{P M N S}$; IH neutrino masses, nonunitary $U_{P M N S}$. We discussed these issues for unflavored leptogenesis regimes, for which $M_{1} \geq 10^{12}$ $\mathrm{GeV}$, where $M_{1}$ is the lightest one of the three heavy righthanded Majorana neutrinos, whose out-of-equilibrium decay produces lepton asymmetry (which in turn can be converted to BAU).

In this work, we have calculated new limits on nonunitarity parameters using latest bounds on CLFV decays and thus predicted values of lightest neutrino mass (Table 2) for both the hierarchies, which is still unknown experimentally. We also have predicted values of CPV phases $-\delta_{C P}$ (Dirac phase) and $\alpha$ and $\beta$ (Majorana phases), which are also unknown so far (Table 3). Though Majorana phases do not affect neutrino oscillation probability, they may affect neutrino mass measurements in $0 \nu \beta \beta$ experiments. Hence the results in this work are important, keeping in view that in future experiments will be endeavoring to measure the values of absolute value of neutrino mass and CP-violating phase $\delta_{C P}$ and $\alpha, \beta$ (Majorana phases). Future measurements related to Dirac CPV phase in neutrino experiments will validate or contradict some of the results presented here. Our analysis in this work only provides a benchmark for consistent works affiliated to model building.

\section{Disclosure}

This paper is a talk presented at the XXII DAE BRNS High Energy Physics Symposium, 12-16 December 2016, Delhi University, India.

\section{Conflicts of Interest}

The authors declare that they have no conflicts of interest.

\section{Acknowledgments}

Gayatri Ghosh and Kalpana Bora would like to thank Debasish Borah for fruitful discussions and suggestions. Gayatri Ghosh would like to acknowledge UGC, Government of India, for providing RFSMS fellowship to her, during which this work was done. Gayatri Ghosh also acknowledges the fruitful discussions carried out on the constraints of the absolute neutrino mass scale sum at the XXII DAE BRNS High Energy Physics Symposium, 12-16 December 2016, Delhi University, India.

\section{References}

[1] A. J. Cuesta, V. Niro, and L. Verde, "Neutrino mass limits: robust information from the power spectrum of galaxy surveys," Astrophysics: Cosmology and Nongalactic Astrophysics, vol. 13, 2016. 
[2] A. M. Baldini et al., "Search for the lepton flavour violating decay $\mu^{+} \longrightarrow e^{+} \gamma$ with the full dataset of the MEG experiment," The European Physical Journal C, vol. 76, no. 8, p. 434, 2016.

[3] I. Esteban, M. C. Gonzalez-Garcia, M. Maltoni, I. MartinezSoler, and T. Schwetz, "Updated fit to three neutrino mixing: exploring the accelerator-reactor complementarity," Journal of High Energy Physics, vol. 2017, p. 87, 2017.

[4] S. Davidson, E. Nardi, and Y. Nir, "Leptogenesis," Physics Reports, vol. 466, no. 4-5, pp. 105-177, 2008.

[5] V. Kuzmin, V. Rubakov, and M. Shaposhnikov, "On the anomalous electroweak baryon number nonconservation in the early universe," Physics Letters B, vol. 155, p. 36, 1985.

[6] A. Abada, S. Davidson, F. Josse-Michaux, M. Losada, and A. Riotto, "Flavour issues in leptogenesis," Journal of Cosmology and Astroparticle Physics, vol. 2006, 2006.

[7] W. Rodejohann, "Non-unitary lepton mixing matrix, leptogenesis and low-energy CP violation," EPL (Europhysics Letters), vol. 88 , no. 5, 2009.

[8] J. A. Casas and A. Ibarra, "Oscillating neutrinos and muon $\rightarrow$ e, gamma," Nuclear Physics B, vol. 618, no. 1-2, pp. 171-204, 2001.

[9] S. Davidson, E. Nardi, and Y. Nir, "Leptogenesis," Physics Reports, vol. 466, no. 105, pp. 105-177, 2008.

[10] A. Sakharov, "Violation of CP invariance, C asymmetry, and baryon asymmetry of the universe," Pisma V Zhurnal Éksperimental'noй i Teoreticheskoŭ Fiziki, vol. 5, no. 32, 1967.

[11] Z. Z. Xing and S. Zhou, "Implications of leptonic unitarity violation at neutrino telescopes," Physics Letters B, vol. 666, no. 2, pp. 166-172, 2008.

[12] M. Malinsky, T. Ohlsson, and H. Zhang, "Nonunitarity effects in a realistic low-scale seesaw model," Physical Review D: Particles, Fields, Gravitation and Cosmology, vol. 79, no. 7, Article ID 073009, 2009.

[13] A. S. Joshipura, E. A. Paschos, and W. Rodejohann, "Leptogenesis in left-right symmetric theories," Nuclear Physics B, vol. 611, pp. 227-238, 2001.

[14] R. Barbieri, P. Creminelli, A. Strumia, and N. Tetradis, "Baryogenesis through leptogenesis," Nuclear Physics B, vol. 575, no. 1-2, pp. 61-77, 2000.

[15] A. Abada, S. Davidson, A. Ibarra, F. Josse-Michaux, M. Losada, and A. Riotto, "Flavour matters in leptogenesis," Journal of High Energy Physics, vol. 2006, 2006.

[16] E. Nardi, Y. Nir, E. Roulet, and J. Racker, "The importance of flavor in leptogenesis," Journal of High Energy Physics, vol. 164, no. 1, pp. 4123-4149, 2006.

[17] P. S. Dev, P. Millington, A. Pilaftsis, and D. Teresi, "Flavour covariant transport equations: an application to resonant leptogenesis," Nuclear Physics B, vol. 886, pp. 569-664, 2014.

[18] K. Bora, G. Ghosh, and D. Dutta, "Octant Degeneracy and Quadrant of Leptonic CPV Phase at Long Baseline," Advances in High Energy Physics, vol. 2016, 11 pages, 2016, [talk presented at Simplicity II, Fermilab Theory Workshop, Fermilab USA, Sep 6- 9, 2016].

[19] M. Borah, D. Borah, and M. K. Das, "Discriminating Majorana neutrino textures in light of the baryon asymmetry," Physical Review D: Particles, Fields, Gravitation and Cosmology, vol. 91, no. 11, Article ID 113008, 2015.

[20] G. Ghosh and K. Bora, "Effects of Leptonic Non-unitarity on Charged Lepton Flavor Violation, Leptogenesis and Lightest Neutrino Mass," in XXII DAE High Energy Physics Symposium, vol. 203 of Springer Proceedings in Physics, pp. 309-311, Springer International Publishing, Cham, Switzerland, 2018.
[21] K. Bora and G. Ghosh, "Charged lepton flavor violation $\mu \rightarrow e \gamma$ in $\mu-\tau$ symmetric SUSY SO(10) mSUGRA, NUHM, NUGM, and NUSM theories and LHC," The European Physical Journal C, vol. 75, no. 428, 2015.

[22] B. D. Fields, P. Molarto, and S. Sarkar, "Big bang nucleosynthesis," in Review of PDG-2014 (Astrophysical Constants and Parameters), 2014. 

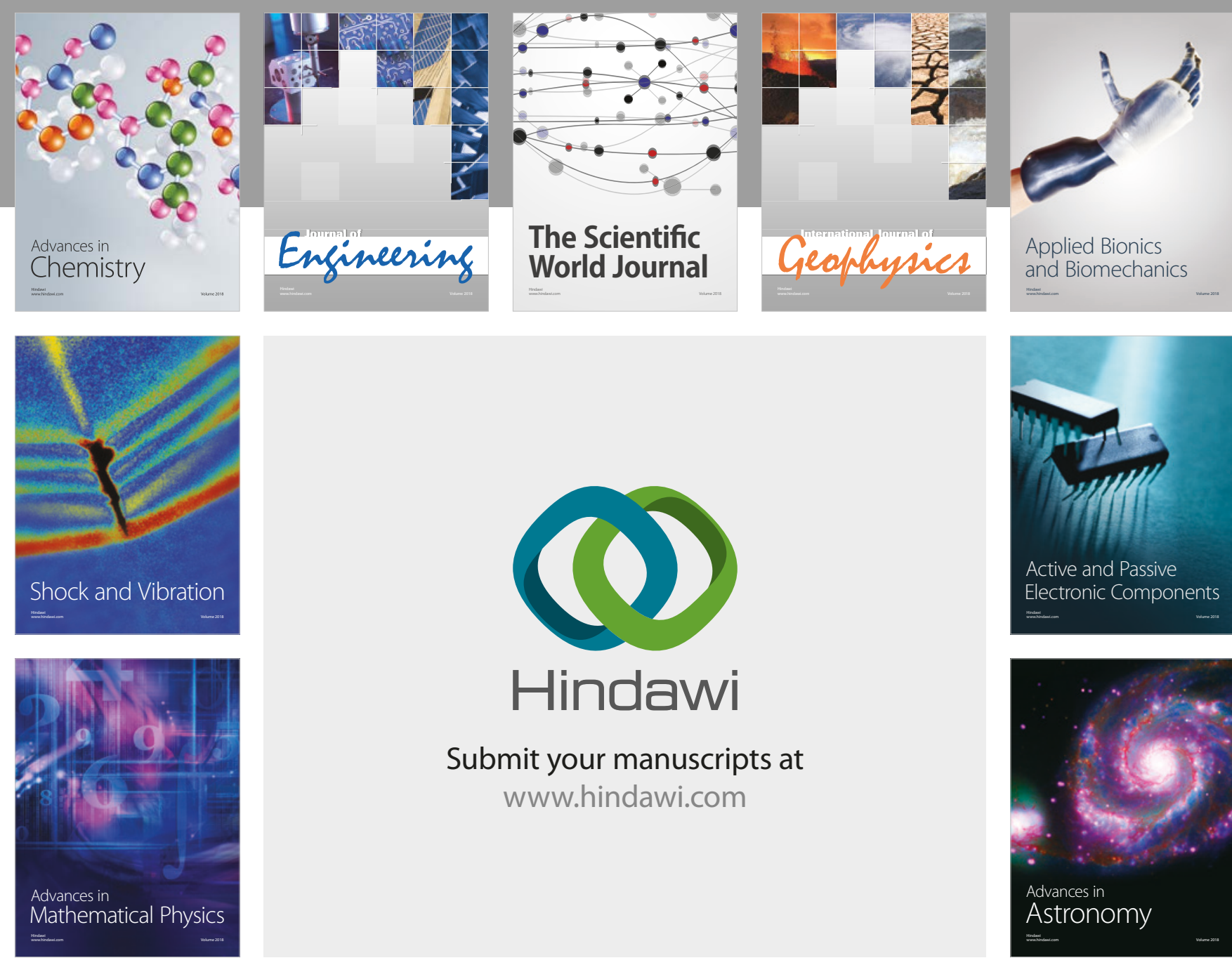

Submit your manuscripts at

www.hindawi.com

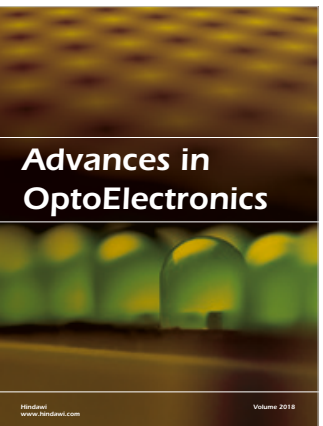

\section{Rotcting Machinery}
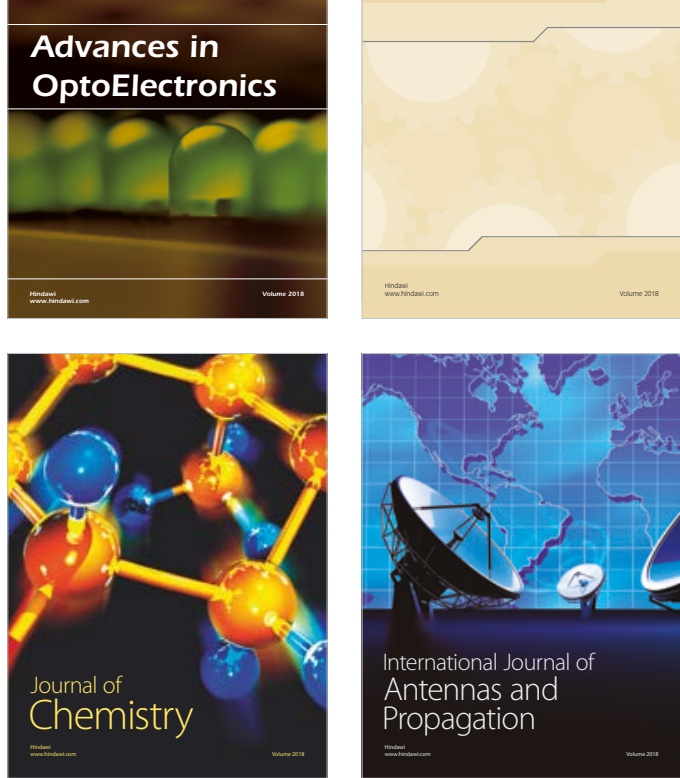

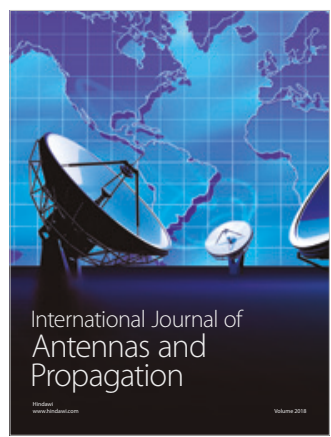

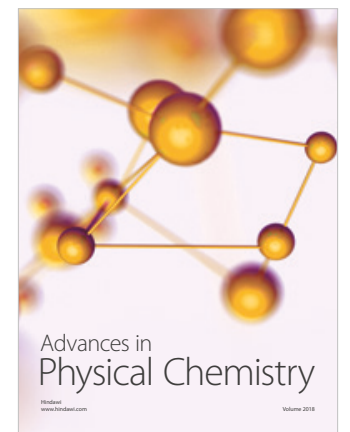

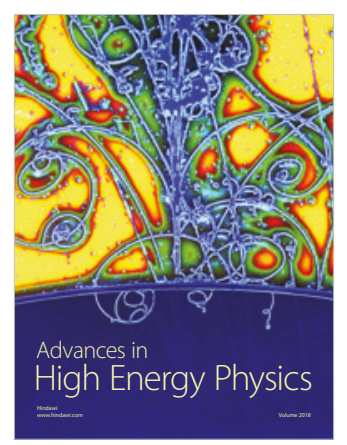

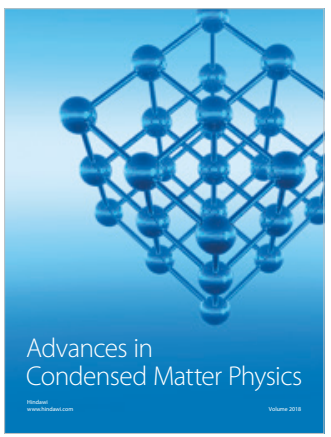

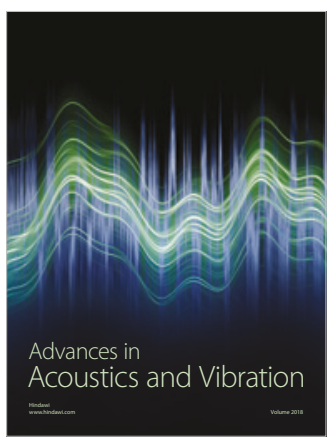

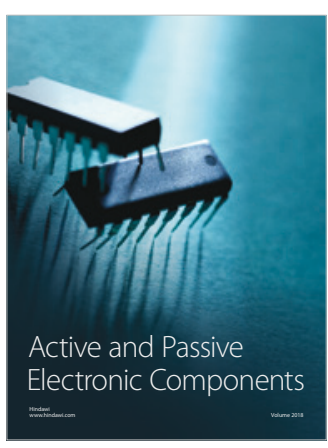
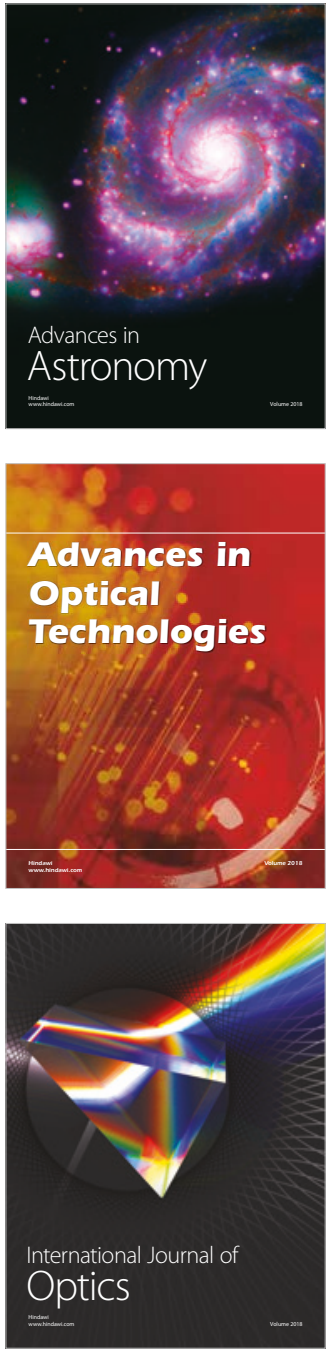